\title{
Criminologie
}

\section{Le construit de l'ethnicité en criminologie}

\section{Mylène Jaccoud}

Volume 36, numéro 2, automne 2003

Le construit de l'ethnicité en criminologie

URI : https://id.erudit.org/iderudit/007863ar

DOI : https://doi.org/10.7202/007863ar

Aller au sommaire du numéro

Éditeur(s)

Les Presses de l'Université de Montréal

ISSN

0316-0041 (imprimé)

1492-1367 (numérique)

Découvrir la revue

Citer ce document

Jaccoud, M. (2003). Le construit de l'ethnicité en criminologie. Criminologie, 36(2), 3-7. https://doi.org/10.7202/007863ar d'utilisation que vous pouvez consulter en ligne.

https://apropos.erudit.org/fr/usagers/politique-dutilisation/ 


\section{Le construit de l'ethnicité en criminologie}

Mylène Jaccoud

Professeure

École de criminologie

Université de Montréal

mylene.jaccoud@umontreal.ca

La criminologie, en tant que savoir à prétention scientifique, est née à la fin du XIX ${ }^{\mathrm{e}}$ siècle. Elle prend naissance dans le mouvement d'émergence des sciences sociales, fascinées par la quantification et la mesure des comportements humains. La science positive du crime et du criminel s'impose dès lors comme l'étude de celui qui contrevient aux normes sociales, mais plus spécifiquement comme l'étude de celui qui représente une menace à la cohésion sociale. Dans les thèses fondatrices de la criminologie, le «criminel» est construit comme fondamentalement différent du non-criminel. Le «passage à l'acte» constitue le critère central de différenciation, mais aussi le symptôme évident d'une anomalie biologique, psychologique ou sociale. Dans un contexte où les effets de l'industrialisation et de la migration croissante (migration urbaine et immigration) requièrent des instruments de gestion et de contrôle des populations reléguées et confinées en marge de la société, la criminologie naissante s'avère sélective : le pauvre, le Noir et l'immigrant sont alors le point de mire d'une «science» qui se doit de participer au maintien de l'ordre social. Science de l'Autre par excellence, un Autre essentialisé par une différence et une infériorité présumées, la criminologie propose des objets interchangeables : passage à l'acte et altérité se superposent, se fondent et se confondent. Le pauvre, le Noir et l'immigrant sont étudiés non seulement pour ce qu'ils font, mais aussi pour ce qu'ils sont. La criminologie participe ainsi de la mise en science d'une frontière entre un Nous collectif défini par sa conformité 
aux normes sociales, par sa «respectabilité» ou par l'invisibilité de ses illégalismes et un Autre «verticalisé» dans la structure sociale, un Autre qu'il convient de contrôler, de surveiller et de normaliser.

Travaillant à partir de populations institutionnalisées, le savoir criminologique s'est érigé sur des fondations partielles en occultant tout questionnement sur les enjeux politiques de la criminalisation des franges sociales les plus vulnérables de la société. Les thèses fondatrices de la criminologie sont remises en cause à partir des années 1960. Un courant critique va rompre avec ces prénotions et ces fondements, pour insister sur le caractère construit de la notion de «crime» et surtout pour dénoncer les prétentions scientifiques d'un savoir s'étant constitué et consolidé à partir de représentations partielles de la délinquance. La rupture paradigmatique qui ébranle les savoirs fondateurs de la criminologie s'opère dans d'autres types de savoirs. Les travaux de Barth ${ }^{1}$ sont, à cet égard, déterminants en anthropologie. Insistant sur la nécessité de dépasser le caractère ontologique de l'ethnicité, Barth suggère de s'intéresser aux processus constituant les frontières entre les différents groupes sociaux. Ces travaux donneront une assise théorique importante au mouvement constructiviste et subjectiviste qui traverse la sociologie des relations ethniques et l'anthropologie contemporaine. L'ethnicité n'est plus envisagée comme une qualité observable, matérialisable et repérable, mais bien comme le produit d'un rapport social entre groupes disposant de ressources et de pouvoirs différenciés. Reconnaître le caractère dynamique, fluide et construit des attributs de l'ethnicité ne veut pas nécessairement dire que celle-ci ne comporte aucune dimension objective. La nationalité, l'immigration (et le statut d'immigrant), les identités revendiquées et attribuées forment des données repérables pour l'observateur. D'ailleurs, les articles présentés dans ce numéro permettent de bien comprendre que le fait de s'interroger sur «le construit de l'ethnicité en criminologie» ne fait pas de l'ethnicité une simple vue de l'esprit; il s'agit plutôt d'une posture critique invitant à intégrer dans l'analyse la diversité des interfaces et des rapports qui structurent les comportements et les identités des acteurs et des institutions. Autrement dit, l'ethnicité constitue une réalité sociologique tant et aussi longtemps que des individus et des groupes s'y réferrent comme univers de sens, que celui-ci soit imposé, attribué ou autodésigné.

1. Barth, F. (1969). Ethnic groups and boundaries. The social organisation of cultural difference. Londres : Allen and Unwin. 
Les premières brèches ouvertes dans le paradigme positiviste en criminologie sont bien illustrées dans l'analyse originale que propose Fabienne Brion d'une note de recherche publiée par Garfinkel en 1949. Celui qui deviendra le chef de file de l'ethnométhodologie compare 673 homicides inter et intraraciaux jugés en Caroline du Nord entre 1930 et 1940. Le classement que Garfinkel élabore en fonction du statut «racial» de l'auteur et de la victime d'homicide amène celui-ci à distinguer quatre groupes : Noir-Blanc, Blanc-Blanc, Noir-Noir et Blanc-Noir, distinctions générant des polarisations particulièrement révélatrices du destin judiciaire des auteurs d'homicide. Dans le couple N-B, le procès prend l'allure d'un rituel et l'acte est conçu comme foncièrement criminel; dans le couple B-B, l'acte est criminel, mais des preuves sont exigées et la responsabilité doit être établie; le couple N-N suscite, comme le souligne Brion, un désintérêt teinté de lassitude, tandis que dans le couple B-N, les justiciers semblent peu enclins à concevoir l'acte comme un crime. Le clivage racial n'est cependant jamais explicitement évoqué par Garfinkel, mais caricaturé par ses couples d'initiales. La note de recherche de Garfinkel, que Brion analyse finement, constitue dès lors un repère historique fascinant pour comprendre l'avènement d'une criminologie déconstruisant le caractère ontologique de l'ethnicité et de la race et dévoilant les prémisses d'une mise à jour des constructions pénales à l'oeuvre dans la mise en rapport des catégories «crime» et «ethnicité».

Le regard sociologique proposé par Laurent Mucchielli sur la délinquance et l'immigration en France est une occasion de mieux connaître l'état de la délinquance des étrangers et celle des jeunes issus de l'immigration. L'analyse sociologique et historique à laquelle L. Mucchielli nous convie procède à plusieurs déconstructions, notamment celles du lien entre délinquance et immigration, des thèses culturalistes mises de l'avant pour expliquer la surdélinquance présumée des immigrants et des étrangers ainsi que des statistiques policières comme reflet fiable de la criminalité. L'auteur souligne la coexistence de deux problématiques distinctes : la délinquance des immigrants et celle des étrangers. Ces problématiques ne sont ni mesurées, ni mesurables de manière identique, tout comme elles ne peuvent être interprétées de la même façon. L. Mucchielli rappelle que les sources statistiques policières fournissent davantage un portrait des activités policières (à l'endroit des étrangers et non des immigrants) que des comportements délinquants. À cet égard, l'auteur souligne comment les tris opérés par les policiers sont fortement conditionnés par les stéréotypes, parlant même d'un «tri ethnique». Par 
contre, et c'est là à notre sens la force de cet article, L. Mucchielli évite le piège du déconstructionnisme absolu : les logiques sélectives des policiers expliquent la surreprésentation des étrangers dans certaines catégories de délits, mais les statistiques policières correspondent, pour d'autres catégories, à des réalités. Dans ces conditions, la surdélinquance observée des étrangers doit essentiellement être interprétée, selon l'auteur, comme une délinquance fortement liée aux conditions de vie.

La construction ethnicisante des conduites délinquantes collectives des jeunes issus des minorités est envisagée par Laurence Tichit à partir d'une revue de littérature américaine. Des travaux de l'École de Chicago en passant par les travaux contemporains, L.Tichit résume les principales thèses proposées pour rendre compte du phénomène des gangs juvéniles, mais surtout pour comprendre comment l'objet «gang de rue» est issu d'un contexte historique et national précis. L'ethnicisation des gangs juvéniles résulte d'une structuration et d'une ségrégation des Noirs et autres minorités aux États-Unis. Par conséquent, les gangs sont le produit d'un rapport social et historique dans lequel les variables « race» et «ethnicité» prennent sens et forme pour les acteurs situés aux deux pôles de la relation. L'article de Tichit illustre très bien, à ce titre, à quel point la perspective constructiviste ne peut être réduite à un processus d'attribution. À cet égard, la grille de Martiniello que Tichit suggère d'utiliser dans l'appréhension de l'ethnicité et dans celle du phénomène des gangs comporte 3 niveaux : 1) le niveau individuel de la dimension subjective de l'ethnicité (sentiment d'appartenance); 2) le niveau groupal de la mobilisation et l'action collective, que Tichit nomme l'«agir ethnique» et 3) le niveau macrosocial des contraintes structurelles qui façonnent les identités et qui assignent les individus à une position déterminée. À partir de l'exemple des gangs juvéniles afro-américains, L. Tichit ouvre la réflexion sur le glissement contemporain de la thématique gang de rue, ethnicité et violence collective de la jeunesse en "problème de sécurité publique», en le ramenant aux facteurs sociopolitiques et au contexte national dans lequel il émerge.

Enfin, Mylène Jaccoud rend compte des résultats d'une recherche portant sur l'analyse de 47 récits d'expérience d'insertion de personnes ciblées par le programme d'accès à l'égalité au Service de police de la Ville de Montréal. L'angle d'approche de l'auteure consiste à évaluer dans quelle mesure les clivages identitaires subsistent, se renforcent, se transforment, s'atténuent ou se fondent dans les interactions qui se tissent entre policiers au sein de l'organisation policière. Les conclusions de cette 
étude nous laissent perplexes quant à la portée des programmes d'accès à l'égalité. D'une part, les frontières identitaires ne sont pas déconstruites et subsistent au sein de l'organisation policière, particulièrement celles qui émanent des rapports entre policiers identifiés aux «minorités visibles» et les «autres»; d'autre part, la diversité incluse dans l'organisation policière semble une diversité de surface puisque, dans certains cas, le service intègre des individus appartenant à des groupes minoritaires qui, en fait, ne se perçoivent pas comme tels. Les attributs «visibles» ou «audibles» intégrés dans l'organisation policière remplissent des fonctions politiques indéniables. Les résultats de cette étude amènent l'auteure à conclure que l'un des objectifs recherchés par les programmes d'accès à l'égalité, notamment celui d'inclure la diversité en espérant transformer les pratiques organisationnelles et, ce faisant, améliorer les rapports avec les communautés ethnoculturelles, reste très limité. 